\title{
The luminous $B[e]$ binary AS 381
}

A. S. Miroshnichenko ${ }^{1,2}$, K. S. Bjorkman ${ }^{1}$, E. L. Chentsov ${ }^{3,4}$, V. G. Klochkova ${ }^{3,4}$, O. V. Ezhkova ${ }^{5,6}$, R. O. Gray ${ }^{7}$, P. García-Lario ${ }^{8}$, J. V. Perea Calderón ${ }^{9}$, R. J. Rudy ${ }^{10}$, D. K. Lynch ${ }^{10}$, S. Mazuk $^{10}$, C. C. Venturini ${ }^{10}$, and R. Puetter ${ }^{11}$

1 Ritter Observatory, Dept. of Physics \& Astronomy, University of Toledo, Toledo, OH 43606-3390, USA

2 Central Astronomical Observatory of the Russian Academy of Sciences at Pulkovo, Saint-Petersburg 196140, Russia

3 Special Astrophysical Observatory of the Russian Academy of Sciences, Karachai-Cirkassian Republic, Nizhnij Arkhyz 369167, Russia

4 Isaac Newton Institute of Chile, SAO Branch, Russia

5 Ulugh Beg Astronomical Institute of the Uzbek Academy of Sciences, Astronomicheskaya 33, Tashkent 700052, Uzbekistan

${ }^{6}$ Issac Newton Institute of Chile, Uzbekistan Branch, Uzbekistan

7 Dept. of Physics and Astronomy, Appalachian State University, Boone, NC 28608, USA

8 ISO Data Centre, Astrophysics Division, Space Science Department of ESA, Villafranca del Castillo, Apartado de Correos 50727, 28080 Madrid, Spain

9 INSA S.A., Villafranca del Castillo, Apartado de Correos 50727, 28080 Madrid, Spain

10 The Aerospace Corp. M2/266, PO Box 92957, Los Angeles, CA 90009, USA

11 Center for Astrophysics and Space Sciences, University of California, San Diego, C-0111, La Jolla, CA 92093, USA

Received 2 November 2001 / Accepted 22 November 2001

\begin{abstract}
We present the results of optical and near-IR spectroscopic and broadband multicolour photometric observations of the emission-line star AS 381. Its properties were found to be similar to those of Be stars with warm dust, a group of galactic objects recently defined by Sheikina et al. (2000). The spectrum of AS 381 indicates the presence of both a hot (early B-type) and a cool (K-type) star in the system. A high interstellar reddening $\left(A_{V} \sim 7 \mathrm{mag}\right)$ suggests that it is located at a distance of $\geq 3 \mathrm{kpc}$, and the companions have luminosity types II or higher. The emission-line profiles indicate that the system is surrounded by a flattened circumstellar envelope, which is viewed close to pole-on. The hot companion is found to be $\sim 2$ mag brighter in the $V$-band and more massive $\left(\sim 20 M_{\odot}\right)$ than the cool one $\left(\sim 7 M_{\odot}\right)$. The strong line emission and position of the companions in the Hertzsprung-Russell diagram indicate that the system is experiencing mass exchange. We suggest that AS 381 is the first B[e] supergiant binary discovered in the Milky Way.
\end{abstract}

Key words. stars: emission-line, Be - stars: individual: AS 381 - techniques: spectroscopic - techniques: photometric

\section{Introduction}

Many early-type emission-line stars were detected in the objective-prism surveys of the past (e.g., MWC, Merrill \& Burwell 1933; AS, Merrill \& Burwell 1950; LS, Stock et al. 1960). These surveys contain thousands of objects, many of which are listed in more than one catalog. In order to summarize the previous findings, Wackerling (1970) compiled a list of 1159 objects found in the above surveys before 1970. Most of them, especially those with very strong emission-line spectra, display a noticeable excess radiation in the infrared (IR) region due to the presence

Send offprint requests to: A. S. Miroshnichenko, e-mail: anatoly@physics.utoledo.edu of circumstellar gas and/or dust. Ground-based IR observations (usually shortward of $10-20 \mu \mathrm{m}$ ) were obtained in the 1970-s for a number of the bright objects, allowing one to study properties of the circumstellar matter. However, much progress in these studies was achieved after the IRAS all-sky survey, which detected about a quarter of a million point-like objects in the region $12-100 \mu \mathrm{m}$. IRAS detected a significant number of Wackerling's objects. Most of those with very strong IR fluxes $(V-[25] \geq$ 8 mag) were identified by Dong \& Hu (1991).

In 1993 we began a study of a subsample of Dong \& Hu's list which mostly contains B-type stars with a steep decrease of IR fluxes longward of $12 \mu \mathrm{m}$ (e.g., Sheikina et al. 2000). These objects seem to form a group with 
similar properties of both the stars and circumstellar matter. Some of them were previously identified as B $[\mathrm{e}]$ stars by Allen \& Swings (1976). Their spectral energy distribution (SED) in the IR region suggests the presence of circumstellar dust, most of which is warmer than $\sim 200 \mathrm{~K}$. This is about an order of magnitude higher than the lowest dust temperature in the envelopes of Herbig Be stars (e.g., Miroshnichenko et al. 1999). Because of this property we refer to the entire group as Be stars with warm dust. These objects display many strong emission lines in their spectra and a few absorption features, which makes studies of the illuminating sources difficult and requires high-resolution observations. They also show noticeable photometric variations in both optical and near-IR region, investigation of which needs long-term observations (e.g., Miroshnichenko 1998). A certain lack of data makes their studies slow and, as a result, their evolutionary state is still unclear (e.g., Lamers et al. 1998).

Our efforts in the study of Be stars with warm dust resulted in a number of publications, where we introduced these remarkable objects and attempted to estimate their physical parameters (see Miroshnichenko 1998 for a recent review and Sheikina et al. 2000). In most cases, high-resolution spectroscopy and multicolour photometric observations were reported for the first time. Since the objects are rather faint $(V \sim 10-14 \mathrm{mag})$, one needs large telescopes to obtain spectra with a reasonable resolution and signal-to-noise ratio. Although they generally share similar overall characteristics, some objects show unique features which deserve special attention. This is why we present them individually or in small groups. Our previous publications dealing with the discussed objects include Miroshnichenko \& Corporon (1999; MWC 342), Miroshnichenko et al. (2000; AS 78 and MWC 657), and Miroshnichenko et al. (2001; HD 85567, Hen3-140 and Hen3-1398).

In this paper we present the first results of our observations of AS 381, another object from Dong \& Hu's (1991) list. It was found in the course of the Mount Wilson survey (Merrill \& Burwell 1950) as an 11.5-mag star with a strong $\mathrm{H} \alpha$ emission. We found no observational results for this object in the literature, except for some IR satellite data (IRAS and MSX) and two near-IR observations discussed below. Here we summarize the data accumulated so far, estimate the star's fundamental parameters, and discuss its evolutionary state.

\section{Observations}

The photometric $U B V R$ (Johnson system) observations of AS 381 were obtained in July-September 2000 at Mt. Maidanak (Uzbekistan) using a 48-cm AZT14 reflector with a standard pulse-counting photometer (Shevchenko 1989). The errors of individual observations, which are presented in Table 1 and in Fig. 1, were as follows: $\sigma U=0.15 \mathrm{mag}, \sigma B=0.06 \mathrm{mag}, \sigma V=0.05 \mathrm{mag}$, and $\sigma R=0.02 \mathrm{mag}$. In total 20 observations were obtained. The object's $U$-band flux was measured only 2

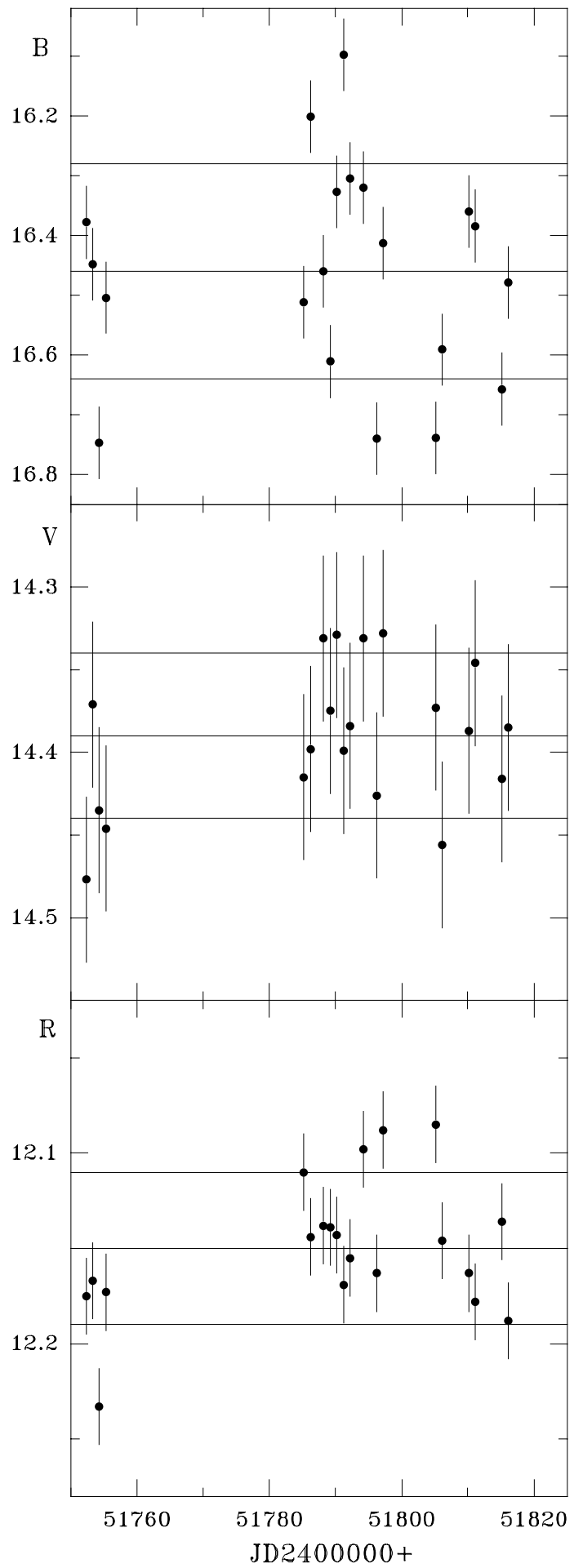

Fig. 1. Optical photometric data for AS 381. Horizontal lines show the mean brightness levels and $1 \sigma$ uncertainty intervals.

times as it is close to the photometer sensitivity threshold. HD 189474 was used as a comparison star, while the instrumental system stability was controlled using other standard stars observed during each night.

On 1999 September 15, 2000 July 24 and 2001 July 27 we obtained $J H K$ photometry of the star at the 1.55-m Carlos Sánchez Telescope (CST), operated by the Instituto de Astrofísica de Canarias at the Spanish 
Table 1. Optical photometry of AS 381.

\begin{tabular}{rcccc}
\hline JD $2451000+$ & $V$ & $U-B$ & $B-V$ & $V-R$ \\
\hline 752.307 & 14.48 & & 1.90 & 2.30 \\
753.308 & 14.37 & 1.47 & 2.08 & 2.20 \\
754.299 & 14.44 & 1.14 & 2.31 & 2.20 \\
755.300 & 14.45 & & 2.06 & 2.27 \\
785.211 & 14.42 & & 2.10 & 2.31 \\
786.200 & 14.40 & & 1.80 & 2.25 \\
788.184 & 14.33 & & 2.13 & 2.19 \\
789.192 & 14.38 & & 2.24 & 2.24 \\
790.187 & 14.33 & & 2.00 & 2.19 \\
791.189 & 14.40 & & 1.70 & 2.23 \\
792.199 & 14.38 & & 1.92 & 2.23 \\
794.190 & 14.33 & & 1.99 & 2.23 \\
796.183 & 14.43 & & 2.31 & 2.26 \\
797.179 & 14.33 & & 2.09 & 2.24 \\
805.156 & 14.37 & & 2.37 & 2.29 \\
806.151 & 14.46 & & 2.14 & 2.31 \\
810.162 & 14.39 & & 1.97 & 2.22 \\
811.162 & 14.35 & & 2.04 & 2.17 \\
815.169 & 14.42 & & 2.24 & 2.28 \\
816.167 & 14.39 & & 2.09 & 2.20 \\
\hline
\end{tabular}

Observatorio del Teide (Tenerife, Spain). We used a CVF infrared spectrophotometer equipped with an InSb photovoltaic detector, operating at the temperature of liquid nitrogen, with a photometric aperture of $15^{\prime \prime}$ and a chopper throw of $30^{\prime \prime}$ in the E-W direction to subtract the contribution from the background sky. The star was observed 4-5 times in each band. The Teide photometric system, as well as its relations with other standard photometric systems is described in Arribas \& Martínez-Roger (1987).

The infrared low-resolution spectroscopic observations of AS 381 were acquired on 2000 July 18 with the 3-m Shane reflector of Lick Observatory and the Aerospace near-infrared imaging spectrograph. The spectrograph, which is described by Rudy et al. (1999), uses two channels to provide wavelength coverage from 0.8 to $2.5 \mu \mathrm{m}$. The blue $(0.8-1.4 \mu \mathrm{m}$, resolution $14 \AA)$ and red $(1.4-2.5 \mu \mathrm{m}$, resolution $36 \AA$ ) spectra were acquired simultaneously. A more detailed technical description of this type of observation is given in Miroshnichenko et al. (2000). A $2^{\prime \prime}$ slit was employed for the observations of AS 381 and its calibrator star HD 191854, a G5 dwarf ( $V=7.45 \mathrm{mag})$. The instrumental response and most of the effects of atmospheric absorption were removed by dividing the spectrum of AS 381 by that of the calibrator. To remove the intrinsic spectrum of the calibrator from this ratio, a model from Kurucz (1994) appropriate to a G5 v star was used.

The averaged results of our near-IR observations and of those found in the literature are presented in Table 2.

A classification-resolution (3.6 $\AA$ per 2 pixels) spectrum of AS 381 was obtained on 1999 October 16/17 using the Gray/Miller Cassegrain spectrograph on the 0.8-m telescope of the Dark Sky Observatory of the Appalachian State University. The spectrum was taken with a 600 lines $\mathrm{mm}^{-1}$ grating in the first order using a
Table 2. Near-IR photometry of AS 381 .

\begin{tabular}{lcccc}
\hline JD 2 $400000+$ & $J$ & $H$ & $K$ & Ref. \\
\hline 47435 & 8.68 & 7.48 & 6.39 & $\mathrm{a}$ \\
50946 & $8.71 \pm 0.02$ & $7.38 \pm 0.02$ & $6.28 \pm 0.01$ & $\mathrm{~b}$ \\
51437.00 & $8.92 \pm 0.03$ & $7.61 \pm 0.02$ & $6.49 \pm 0.01$ & CST \\
51744.81 & 8.98 & 7.89 & 6.82 & Lick \\
51750.51 & $9.23 \pm 0.04$ & $7.96 \pm 0.04$ & $6.90 \pm 0.02$ & CST \\
52118.52 & $8.94 \pm 0.07$ & $7.68 \pm 0.04$ & $6.58 \pm 0.05$ & CST \\
\hline
\end{tabular}

${ }^{\text {a }}$ Data from Aaronson et al. (1990).

b Data from the 2MASS survey (Skrutskie et al. 1997).

$1024 \times 1024$ Tektronics thinned, back-illuminated CCD. The spectral range is $3800-5600 \AA$. The spectrum was reduced using standard methods under IRAF ${ }^{1}$.

A high-resolution spectrum of AS 381 was obtained on 2000 July 12 at the 6-m telescope of the Special Astrophysical Observatory (SAO) of the Russian Academy of Sciences with the échelle-spectrometer PFES (Panchuk et al. 1998) and a $1140 \times 1170$ pixel CCD detector. The spectral range was $4577-7820 \AA$ with the mean $R \sim 15000$. The spectrum was reduced using standard methods under MIDAS.

\section{Results}

\subsection{Literature data}

AS 381 is located in the south-western part of Cygnus, very close to the galactic plane $\left(b=0.6^{\circ}\right)$. There is only one more object in the $5^{\prime}$ vicinity of AS 381 listed in the SIMBAD database. This is a carbon star, cya 22 (star No. 22 in the Cygnus region "a"), discovered by Aaronson et al. (1990). These authors conducted an objective prism survey aiming at finding carbon stars. For most of the detected candidates they obtained high-resolution spectra near the $\mathrm{C}_{2}$ Swan band head at $5636 \AA$ and near-IR photometry in the $J H K$ bands. No spectral information was reported for cya 22 in this paper, while its JHK-magnitudes turned out to be close to our data for AS 381. According to Aaronson et al., cya 22 is located $5^{\prime \prime}$ from AS 381, that is within the uncertainties of their positional measurements. Since the accuracy of the AS 381 position listed in the AS catalog is low, we used recent determinations from the MSX (Egan et al. 1999) and 2MASS (Skrutskie et al. 1997) surveys (RA $20^{\mathrm{h}} 06^{\mathrm{m}} 39^{\mathrm{s}} .9$, Dec $\left.+33^{\circ} 14^{\prime} 27^{\prime \prime}, 2000.0\right)$. This coincidence raised a question about the classification of cya22 as a carbon star. Both the MSX and 2MASS survey revealed only one source in a $1^{\prime}$ vicinity of the quoted coordinates. Since there is no spectral confirmation of the carbon type nature of cya 22, we conclude that Aaronson et al. detected near-IR radiation of AS 381.

${ }^{1}$ IRAF is distributed by the National Optical Astronomy Observatories, which are operated by the Association of Universities for Research in Astronomy, Inc., under contract with the National Science Foundation. 
The appearance of AS 381 in Dong \& Hu's (1991) list prompted Thé et al. (1994) to include it in their cata$\log$ of the Herbig Ae/Be stellar group objects, which contained 287 early-type emission-line stars. They are potential candidates to pre-main-sequence intermediate-mass $\left(2-10 M_{\odot}\right)$ stars, although a fraction of objects at later evolutionary stages, such as LBVs and B[e] supergiants, has been recognized in this list.

Additionally to the IRAS detection, AS $381=$ IRAS $20047+3305$, was also detected by MSX (MSX5-G070.5812+00.5721). The non colour-corrected IRAS fluxes refined using the ADDSCAN procedure (coaddition of all IRAS scans; see Weaver \& Jones 1992) along with those from the MSX survey are listed in Table 3. The source was not detected by IRAS in the $100 \mu \mathrm{m}$ band. Summarizing the literature search for data on AS 381, we can only say that it was known as an emission-line star associated with a strong IR source. No information about its nature or evolutionary state could be obtained from the data available prior 1999.

\subsection{Photometric variations and spectral energy distribution}

Our optical photometric observations were obtained within a 63-day period and revealed no significant brightness variations. The mean brightnesses are as follows: $\bar{U}=17.9 \pm 0.1, \bar{B}=16.46 \pm 0.18, \bar{V}=14.39 \pm 0.05$, $\bar{R}=12.15 \pm 0.04$. Their uncertainties are within $3 \sigma$ observational errors in each band. Our 2 measurements in the $U$-band turned out to be very close to each other, which is most likely a coincidence because of a low signal-tonoise ratio in this band. Therefore, we consider this result as a rough estimate of the $U$-band brightness of AS 381, and show it in Fig. 2 only for reference. One can see that the star is very reddened and is much fainter than it was reported in the AS catalog $\left(m_{V}=11.5 \mathrm{mag}\right)$. This difference might imply that AS 381 was brighter fifty years ago, although the photometric system of this catalog is uncertain.

The near-IR data collected in Table 2 show that the variations of AS 381 in this spectral region significantly exceed the observational accuracy and possible systematic differences of the instrumental systems. The latter is unlikely to exceed $0.1 \mathrm{mag}$. The character of these variations and the connection to the optical changes are not clear yet and require further observations. However, the data from Table 2 indicate that the color changes are much smaller than the brightness variations $(\overline{J-H}=1.27 \pm 0.05$, $\overline{H-K}=1.10 \pm 0.02)$.

A similar amplitude of the near-IR brightness variations was detected in some Be stars with warm dust, such as MWC 342 (Miroshnichenko \& Corporon 1999) and MWC 657 (Miroshnichenko et al. 2000). The IRAS fluxes are about 20 per cent above the MSX fluxes (obtained in 1983 and 1996, respectively) at close wavelengths (12 and $25 \mu \mathrm{m})$. This difference may be part of the same process, that is responsible for the near-IR variability.

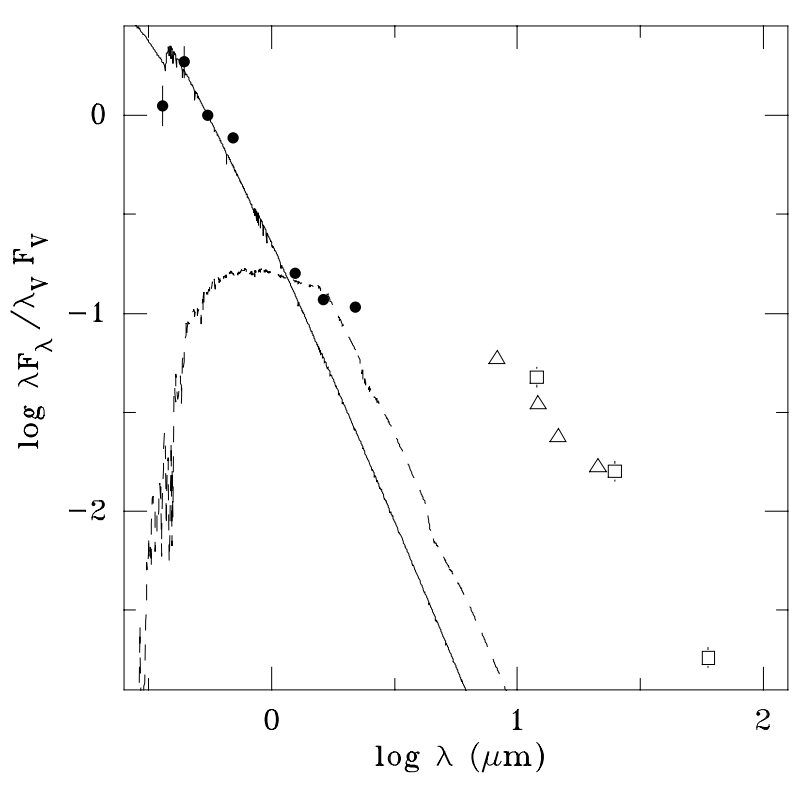

Fig. 2. The SED of AS 381. The solid line represents the synthetic spectrum for $T_{\text {eff }}=25000 \mathrm{~K}$ and $\log g=2.0$, the dashed line shows the synthetic spectrum for $T_{\text {eff }}=4500 \mathrm{~K}$ and $\log g=1.0$ from Kurucz (1994). The Johnson UBVRJHK band fluxes are shown by filled circles, the IRAS data by open squares, the MSX data by open triangles. A reddening of $E_{B-V}=2.4 \mathrm{mag}$ and $V=14.39 \mathrm{mag}$ was assumed. The cool companion is $2.2 \mathrm{mag}$ fainter in the $V$-band.

Thus, the observations reported here along with other available photometric data constrained the SED of AS 381 (see Fig. 2) and showed that its optical brightness is stable on a time scale of months, while it is variable in the IR region on a time scale of years. If we assume that a reddened early B-type star dominates the SED in the visual region (which will be shown in the next section), then the total $V$-band extinction $\left(A_{V}\right)$ turned out to be about $7.4 \mathrm{mag}$ $\left(E_{B-V}=2.4 \pm 0.2 \mathrm{mag}\right)$. Even in this extreme case, the IR excess is significant. Overall, the SED of AS 381 is very similar to those of other Be stars with warm dust. The IR excess is too strong to be explained by free-free radiation of the circumstellar matter and requires the presence of dust in the immediate vicinity of the star.

The photometric data we collected for stars in a region of $\sim 1^{\circ}$ around AS 381 show a smooth increase of $A_{V}$ up to only $2.5 \mathrm{mag}$ within 5-6 kpc from the Sun (Fig. 3a). This result implies that either AS 381 is a very distant object or a significant part of its reddening is circumstellar.

\subsection{Spectrum}

The high-resolution SAO spectrum of AS 381 contains many emission lines, about a dozen strong diffuse interstellar bands (DIBs), and nearly 20 absorption features in its red part. The Balmer lines are extremely strong and narrow (full width at half maximium, $F W H M \sim 110 \mathrm{~km} \mathrm{~s}^{-1}$; see Fig. 4). Their profiles contain neither P Cyg-type absorption features nor broad wings. Other permitted transitions observed (He I, Fe II, Si II) have similar profiles, 
Table 3. Satellite IR photometry of AS 381.

\begin{tabular}{lcccc}
\hline IRAS & $12 \mu \mathrm{m}$ & $25 \mu \mathrm{m}$ & $60 \mu \mathrm{m}$ & $100 \mu \mathrm{m}$ \\
\hline $20047+3305$ & $5.46 \pm 0.01$ & $4.18 \pm 0.05$ & $1.23 \pm 0.20$ & \\
\hline MSX & $8.28 \mu \mathrm{m}$ & $12.13 \mu \mathrm{m}$ & $14.65 \mu \mathrm{m}$ & $21.34 \mu \mathrm{m}$ \\
\hline $5 \mathrm{C}-\mathrm{G} 070.5812+00.5721$ & $4.45 \pm 0.22$ & $4.09 \pm 0.17$ & $3.56 \pm 0.16$ & $3.66 \pm 0.34$ \\
\hline
\end{tabular}

The fluxes are given in Janskys. AS 381 was not detected by IRAS at $100 \mu \mathrm{m}$.
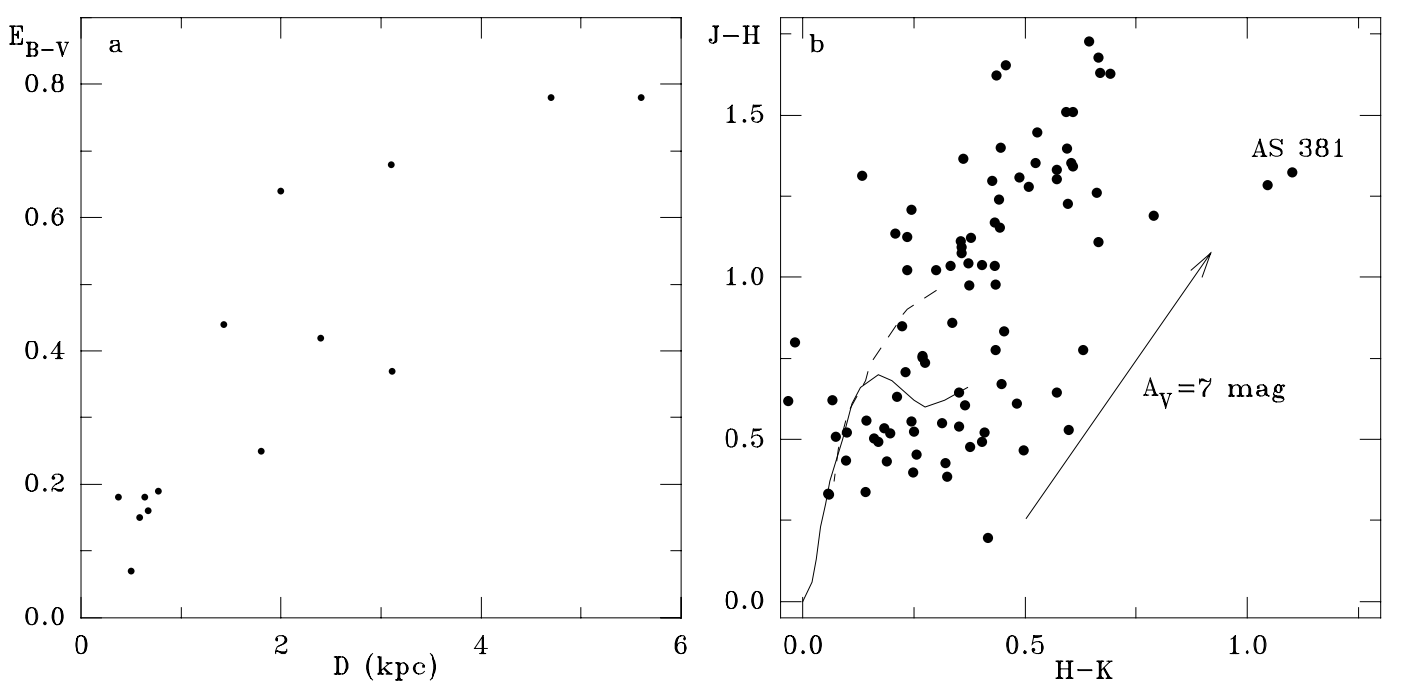

Fig. 3. Photometric properties of stars close to AS 381. a) The interstellar extinction law derived from $U B V$ photometry of stars from a $1^{\circ}$ region around AS 381. b) $J H K$ data for stars from a $3^{\prime}$ region around AS 381 from the 2 MASS survey. The interstellar reddening vector corresponding to $A_{V}=7 \mathrm{mag}$ is shown by the arrowhead line. The intrinsic color-indices for dwarfs are shown by a solid line, those for giants by a dashed line.

which are even narrower $\left(F W H M \sim 70 \pm 10 \mathrm{~km} \mathrm{~s}^{-1}\right)$. We also detected 3 strong emission lines of $[\mathrm{O}$ III] at 4363 , 4959 , and $5007 \AA$. They have the same average $F W H M$ as the permitted lines. The forbidden neutral oxygen lines at 6300 and $6363 \AA$ observed in emission are most likely formed in the object's envelope, but can be telluric in part. The DIBs are strong, which is consistent with the large photometric reddening. For example, the equivalent width of the strongest DIB at $5780 \AA(1.0 \AA)$ corresponds to $E_{B-V} \sim 1.8-2.0 \mathrm{mag}$ (Herbig 1993).

The sodium $\mathrm{D}_{1,2}$ lines consist of two absorption components. One of them is deep and has interstellar origin, while the other one is very weak and most likely circumstellar, showing nearly the same $R V$ as that of the emission lines. The mean $R V$ of the $\mathrm{Na}$ I $\mathrm{D}$ interstellar components is $-17 \mathrm{~km} \mathrm{~s}^{-1}$, while that of the DIBs is $-20 \pm 1 \mathrm{~km} \mathrm{~s}^{-1}$.

The absorption features detected in the red part of the SAO spectrum (5800-7800 $\AA$ ) are neither telluric nor expected from an early-type star. Such lines are usually observed in the spectra of cool stars. Using this suggestion, we identified 23 absorption lines with central intensities between 0.7 and 0.9 (in continuum units) as those of neutral metals. Their $R V \mathrm{~s}$ vary from -10 and $-35 \mathrm{~km} \mathrm{~s}^{-1}$ with an average value of $-22 \pm 3 \mathrm{~km} \mathrm{~s}^{-1}$. Figure $4 \mathrm{f}$ shows that the absorption part of the AS 381 spectrum is qualitatively similar to that of Arcturus.

The mean radial velocity $(R V)$ of 44 detected permitted emission lines is $-40 \pm 1 \mathrm{~km} \mathrm{~s}^{-1}$. At the same time, one can distinguish 3 different groups among the 9 detected forbidden lines. The [O III] lines have an extreme $R V$ of $-48 \pm 1 \mathrm{~km} \mathrm{~s}^{-1}$. The $R V$ of the [Ni II] and [S II] lines is $-35 \pm 3 \mathrm{~km} \mathrm{~s}^{-1}$ and is close to that of the permitted lines. The [O I] lines have a mean $R V\left(-24 \pm 1 \mathrm{~km} \mathrm{~s}^{-1}\right)$ close to that of the absorption lines. This difference is beyond the measurement uncertainties and probably reflects different line formation regions in the circumstellar envelope.

The DSO spectrum, obtained in the bluer range nearly a year before (JD 2451469), shows the same strong and narrow emissions in the overlapping region. Although the spectrum has a low signal-to-noise ratio because of the star's faintness, it demonstrates a relative stability of the line content over the period of the dramatic change in the near-IR.

The near-IR spectrum of AS 381 contains emission lines of neutral hydrogen, carbon, oxygen, and helium as well as of singly ionized calcium and iron (Fig. 5). The strongest emission line identified in this spectrum is that 

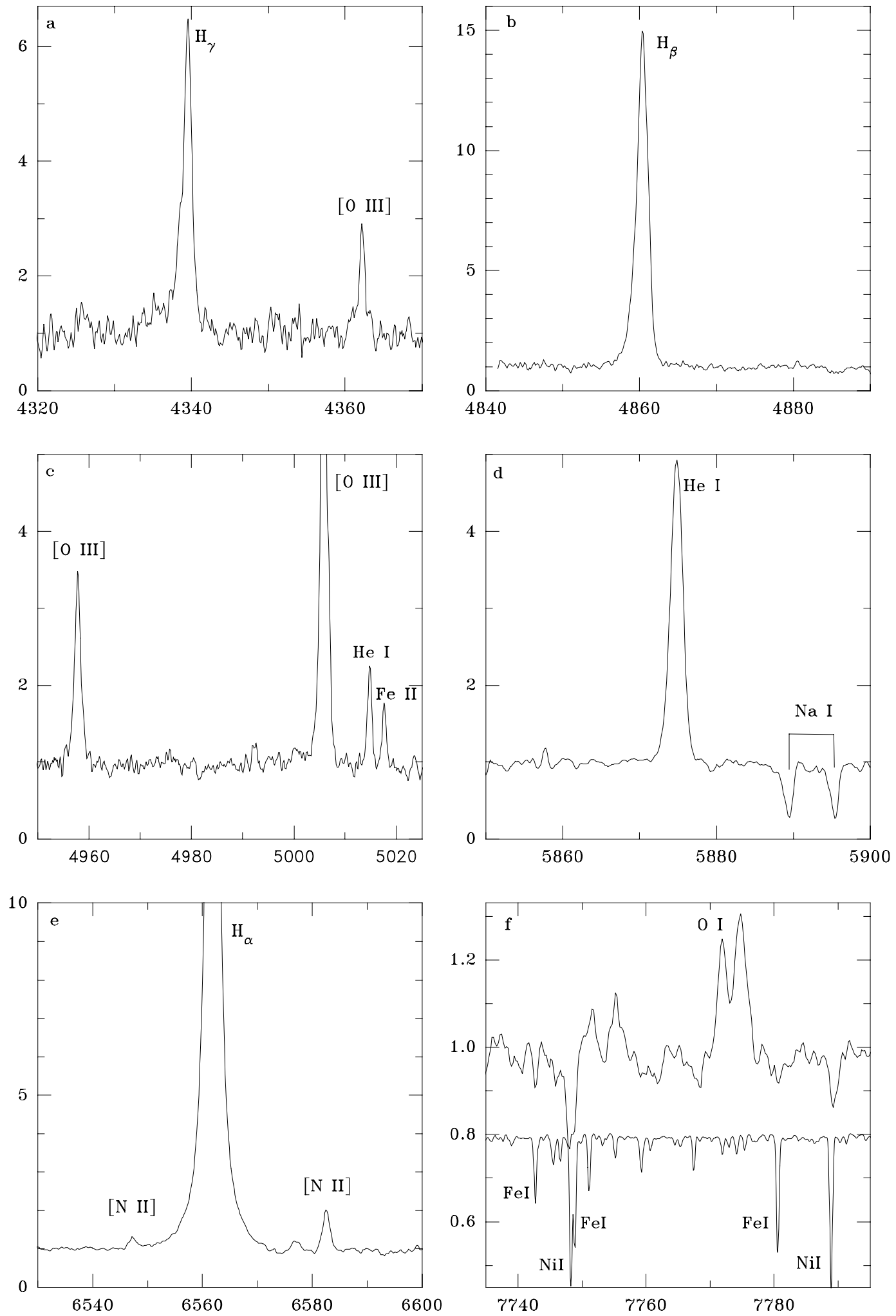

Fig. 4. Parts of the high-resolution SAO spectrum of AS 381. A part of the Arcturus spectrum from Hinkle et al. (2000) is shown in panel f) for comparison (the lower spectrum). Wavelengths are given in $\AA$ while the intensities are normalized to the continuum.

of He I $1.083 \mu \mathrm{m}$, although Paschen lines are strong too. The IR triplet of Ca II has an averaged intensity of 1.2 of the continuum flux. A similar strength is observed in the spectra of other Be stars with warm dust, such as AS 78 and MWC 657. The Brackett lines are in emission, but are much weaker than the Paschen lines. In general, the emission line profiles (not resolved) look similar to those in the optical region. However, the relative strength of the 

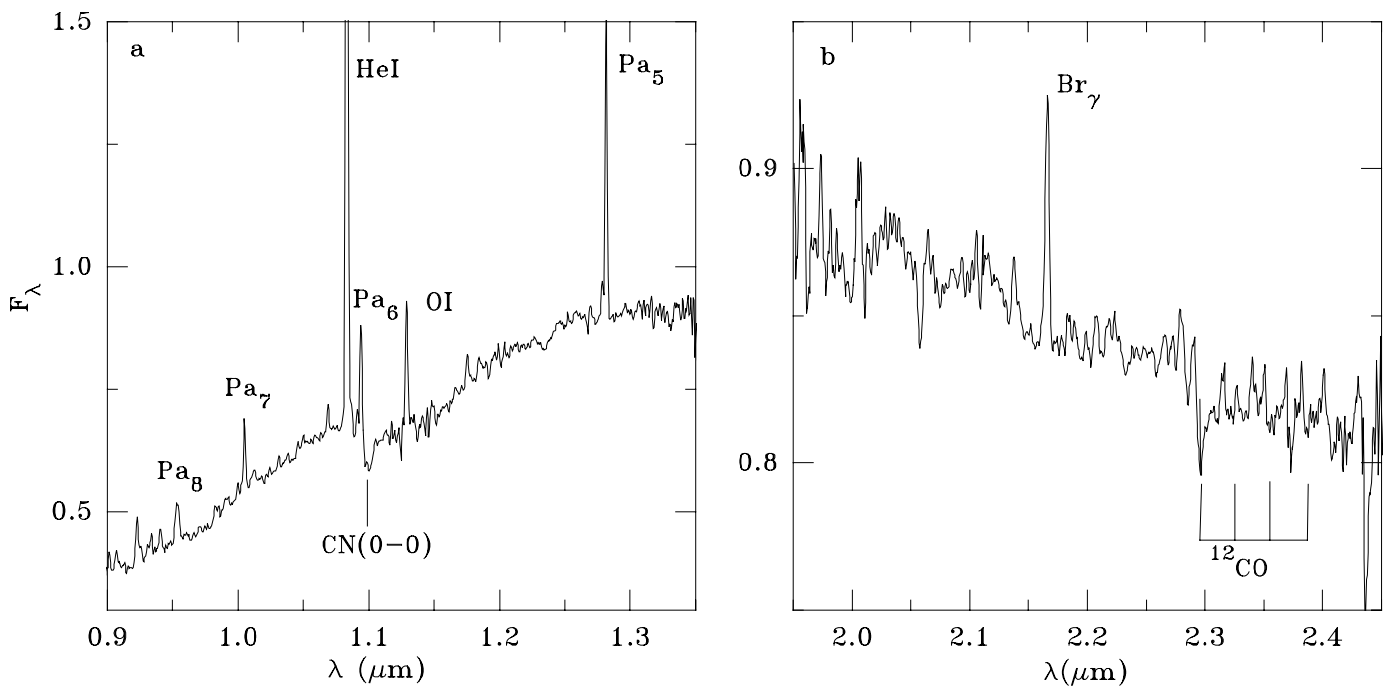

Fig. 5. Parts of the low-resolution Lick near-IR spectrum of AS 381. Wavelengths are given in microns, while the fluxes are given in units of $10^{-16} \mathrm{~W} \mathrm{~cm}^{-2} \mu \mathrm{m}^{-1}$.

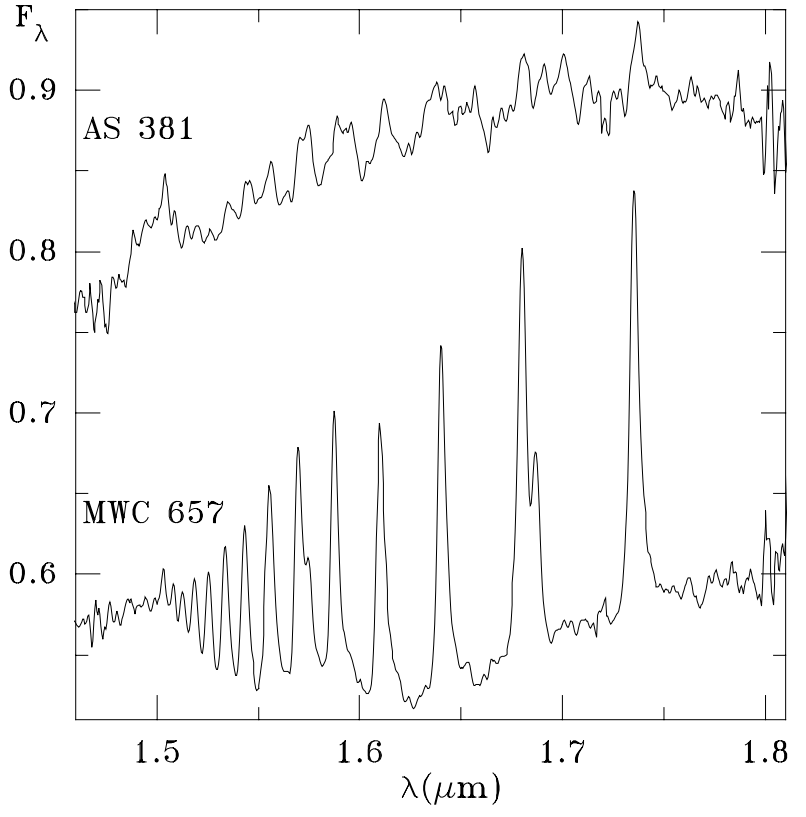

Fig. 6. The $H$-band Lick spectrum of AS 381. The wavelengths and flux units are the same as in Fig. 5. The spectrum of MWC 657, obtained at Lick in July 2000 with the same equipment, is shown for comparison. It is shifted down by $0.2 \times 10^{-16} \mathrm{~W} \mathrm{~cm}^{-2} \mu \mathrm{m}^{-1}$.

optical and infrared lines in the spectrum of AS 381 is much higher than that in MWC 657 (Fig. 6), which has a similar optical spectrum (Miroshnichenko et al. 2000).

The flux ratio of the $\mathrm{O}$ I lines at 8446,11287 , and $13164 \AA$ can be used for an independent interstellar reddening estimate by means of the method suggested by Rudy et al. (1991). This method assumes that an equal number of photons are generated in the 8446 and $11287 \AA$ lines by the $\mathrm{Ly}_{\beta}$ fluorescence mechanism, but corrects this ratio, by using the $13164 \AA$ line, for the additional photons generated in the $8446 \AA$ line by continuum fluorescence.
The flux ratio observed in the spectrum of AS 381 gives $E_{B-V}=2.3 \pm 0.3 \mathrm{mag}$, which agrees with the above photometric estimate.

There is a continuum jump at $1.1 \mu \mathrm{m}$ (see Fig. 5) which seems to be real, as it is not present in the spectra of other objects observed during the same run. This feature is not seen in hot and dwarf stars, but appears in the spectra of stars later than G5 of luminosity types I-III (Wallace et al. 2000 ) and is attributed to the $\mathrm{CN} 0-0$ band. Additionally, we detected an absorption feature at $2.29 \mu \mathrm{m}$ which is most likely due to the $\mathrm{CO} 2-0$ band (Fig. $5 \mathrm{~b}$ ). Other $\mathrm{CO}$ bands, usually observed in this spectral region, are not clearly seen.

The list of lines identified in the spectrum of AS 381 with the help of a catalogue by Coluzzi (1993) is presented in Appendices A (optical range) and B (near-IR range).

\section{Discussion}

The photometric and spectral properties of AS 381 described above show that it is a peculiar and complex object. The presence of the strong hydrogen and He I lines in emission and the absence of He II lines suggest an early-B (B1 \pm 1$)$ spectral type for the star, illuminating the circumstellar matter. The narrow emission line profiles as well as the absence of absorption components and broad wings in them imply two possibilities. First, the circumstellar geometry is disk-like, and the disk is viewed at a large inclination angle (probably close to pole-on). Second, an additional source of continuum, veiling the line wings and absorption components, is present. This source could have either a stellar (radiation of a companion) or circumstellar (free-free and free-bound radiation) origin. Both mentioned mechanisms can be present at the same time, too.

Our detection of absorption lines of neutral metals and molecular bands in the red and near-IR spectrum of AS 381 suggests the presence of a late-type stellar companion. Since no molecular bands are seen in the optical 
spectrum, the spectral type of such a companion should have a spectral type earlier than M0. A later spectral type is unlikely, because M-stars have prominent absorption bands at $\lambda \lambda 0.7-1.0 \mu \mathrm{m}$, which we would detect in our red or near-IR spectra despite the companions' brightness difference. As it is seen from Fig. 4f, the spectrum of AS 381 near $\lambda 7800 \AA$ resembles that of Arcturus, although the AS 381 lines are weaker probably due to the contributions from the hot companion and circumstellar matter. This suggests that the cool companion is most likely a K-type star, because these lines are intrinsically weak in G-type stars and would be almost undetectable in the described situation. Nevertheless, further highresolution spectroscopic observations redward of $\sim 5000 \AA$ with higher signal-to-noise ratios are needed to constrain the cool companion's properties. Direct comparison with the spectra of standard stars seems to be premature.

The CN 0-0 band and CO bands at 2.0-2.3 $\mu \mathrm{m}$ are seen in the spectra of late-G through M-type stars (Wallace \& Hinkle 1997) becoming stronger as the temperature decreases. Our clear detection of only the CO 2-0 band also suggests a not very late spectral type for the companion. The fact that the $\mathrm{CO}$ bands are weak in the spectrum of AS 381 (see Fig. 5b) might be partly explained by the thermal continuum veiling. We estimated a lower limit for the latter of $\sim 50 \%$ in the $K$-band (amount of the thermal flux over that of the late-type companion) assuming no thermal contribution in the $J$ - and $H$-band fluxes, where it cannot be large since we detected the $\mathrm{CN}$ band. This estimate shows that the detected $\mathrm{CO}$ band cannot be intrinsically as strong as that in the M-type spectra $(\sim 50 \%$ of the continuum). Moreover, our resolution is enough to detect other $\mathrm{CO}$ bands if they were strong $(\sim 8$ resolution elements between the 4 bands in the region $2.29-2.38 \mu \mathrm{m}$ ). Thus, both our red and near-IR spectra suggest that the late-type companion is most likely a K-type star.

A K-type spectrum in combination with a B-type spectrum can qualitatively explain the observed SED between the $B$ - and $H$-band (see Fig. 2). However, the SED fitting is complicated by uncertain contributions from both gaseous and dusty components of the circumstellar envelope. The latter also affects the interstellar reddening estimate. Nevertheless according to our preliminary calculations (similar to those described by Waters et al. 1987 for Be stars), the circumstellar reddening due to freefree and free-bound continuum can be of the order of $E_{B-V}^{\mathrm{CS}} \sim 0.3 \mathrm{mag}$. This suggests that the bulk of the observed reddening is interstellar and the line profile veiling is not strong.

A similar suggestion about the system's stellar components was made for another Be star with warm dust, MWC 623 (Zickgraf \& Stahl 1989). In the spectrum of this object many absorption lines from a late-type companion are clearly observed at $\lambda \geq 5000 \AA$. In AS 381 such lines are only seen redward of $\sim 5800 \AA$. This points to a different companions brightness ratio. The hot companion of AS 381 should be brighter than the cool one in the visual region (see Fig. 2), while the situation is reversed in MWC 623. If this is the case for AS 381, then the $B-V$ color-index would not be affected by the cool companion. Hence, most of the observed reddening would be interstellar, suggesting a large distance to the object.

We can put some constraints on the distance towards AS 381 using our $R V$ measurements. Since the system is most likely viewed close to pole-on, the orbital motion does not noticeably affect its $R V$. Unfortunately, models for the galactic rotation (e.g., Dubath et al. 1988) do not agree with the observed $R V$ distribution in the direction of AS 381. The latter just shows that stars with more negative $R V \mathrm{~s}$ are located further away from the Sun but does not match the observed values. Early-type supergiants within $1^{\circ}$ from the object at $D=1-3 \mathrm{kpc}$ have $R V \mathrm{~s}$ ranging from +5 to $-30 \mathrm{~km} \mathrm{~s}^{-1}$, while those belonging to the association Cyg OB3 $(D \sim 2.4 \mathrm{kpc})$ have $R V$ s between -5 and $-25 \mathrm{~km} \mathrm{~s}^{-1}$ (Humphreys 1978). Since closer stars from this region have $R V \mathrm{~s} \geq-10 \mathrm{~km} \mathrm{~s}^{-1}$, the observed $R V$ of AS 381 (in agreement with the large reddening) suggests a large distance. Ignoring possible circumstellar reddening and the gaseous continuum contribution, one can calculate an upper limit for a distance toward AS 381 ( $D=6.4 \mathrm{kpc}$ ) assuming that the object's luminosity does not exceed the Humphreys-Davidson limit. In this case both components need to be supergiants.

A rough estimate of the distance lower limit $(D \sim$ $1.5 \mathrm{kpc}$ ) can be derived under the assumptions that the hot companion is a B1 $\mathrm{V}$ star, it is about 1-2 mag brighter in the $V$-band than the cool companion, and the interstellar reddening is $\sim 7 \mathrm{mag}$. However, it is unlikely that the distance toward AS 381 is less than $\sim 3 \mathrm{kpc}$ (the distance where the interstellar extinction law changes its slope), because luminous stars in its vicinity, located closer to the Sun, are not significantly reddened (see Fig. 3a). It is also the reddest object in the 2MASS survey of its $3^{\prime}$ vicinity (Fig. 3b). The only other star close to its position in the $J-H$ vs. $H-K$ diagram is $\sim 8$ mag fainter. Furthermore, the $\mathrm{CN}$ band at $1.1 \mu \mathrm{m}$ is not seen in the spectra of dwarf stars, implying that the cool companion is at least a giant.

The pole-on orientation of a disk-like envelope may explain the line profile shapes. If it is optically thick in continuum, such an orientation also produce a much stronger continuum in both the optical and near-IR region than in the edge-on case. This, along with the cool companion contribution, may account for the weakness of the Brackett emission lines in AS 381 in comparison with those in MWC 657 (see Fig. 6), whose double-peaked profiles of the optical emission lines strongly suggest that its envelope is viewed nearly edge-on (see Miroshnichenko et al. 2000).

Let us now estimate the physical parameters of the companions. As seen from Fig. 2, the short-wavelength part of the observed SED can be fitted by radiation of an early-B and a K-type star with a brightness ratio of $\sim 2$ mag in the $V$-band. This ratio corresponds to a luminosity difference of $\sim 20$ (with the bolometric corrections of -2 and -1 mag for the hot and cool star, respectively, Miroshnichenko 1997). Therefore, the cool companion is 


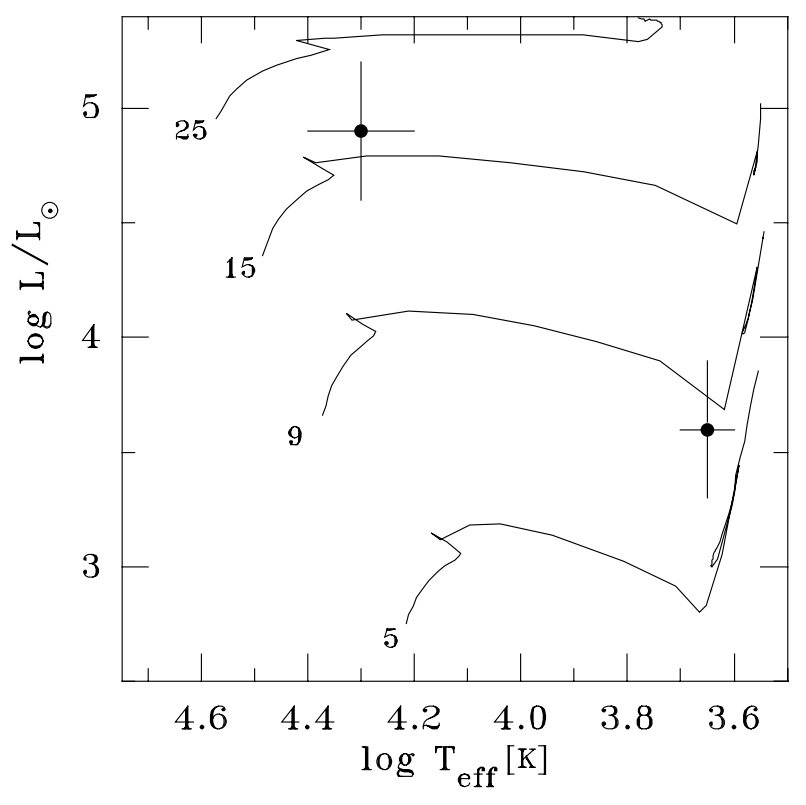

Fig. 7. $H-R$ diagramme with the locations of the hot and cool companion of AS 381. Evolutionary tracks are from Schaller et al. (1992) with initial masses (in solar units) shown near corresponding tracks.

less massive than the hot one. This makes AS 381 similar to another remarkable $\mathrm{B}+\mathrm{K}$ binary, AX Mon, which is an interacting system with a period of 232 days (Elias et al. 1997). At a reasonable distance $D=4 \pm 1 \mathrm{kpc}$, the companions would have $\log L / L_{\odot}=4.9 \pm 0.2$ (B-star) and $3.6 \pm 0.2$ (K-star) which correspond to evolutionary masses of $19 \pm 3$ and $7 \pm 2 M_{\odot}$, respectively (Schaller et al. 1992, see Fig. 7). If the companions evolved as single stars, their ages would be very different. Hence, the system most likely experienced mass exchange. Since AS 381 shows a much stronger emission-line spectrum than AX Mon, this might suggest a stronger ongoing mass transfer between its companions. One might expect an orbital period for AS 381 to be comparable to or smaller than that of AX Mon with a minimum of $\sim 30$ days (based on rough estimates of the companions' masses and radii). However, it might be difficult to detect $R V$ variations because of a small inclination angle of the system's rotation axis to the line of sight, as discussed above.

The above consideration allows us to discuss the system's evolutionary state. It is obviously an evolved object that consists of massive stars. The circumstellar dust may be formed in the wind of either one or both components. The steep decrease in the far-IR flux, seen in the IRAS data, suggests a lack of cool and distant dust. This probably implies that the system is still evolving redward from the main-sequence and is too young to produce a pronounced SED peak at $25-60 \mu \mathrm{m}$, which is observed in the IRAS data for post-AGB stars or LBVs (more arguments against the post-AGB nature of Be stars with warm dust are considered in Miroshnichenko et al. 2000). The presence of forbidden lines in the spectrum of AS 381 allows us to classify it as a B[e] supergiant. The forbidden line velocity stratification is suggestive of a complex structure of the low density regions of the envelope. Since the late-type component is brighter than the early-type one in the nearIR, the observed photometric variations may be due to its pulsational activity. Density changes in the dusty envelope due to sporadic enhancements of the mass loss cannot be excluded either. Another possibility is occultations of a part of the near-IR emitting circumstellar matter by the hot companion, if the stars are close enough to each other. These suggestions still need to be confirmed by follow-up optical and near-IR photometry.

Overall, the observed spectral features of the cool star and the companions' brightness ratio suggest that the system consists of a B-type star and a fainter K-type star, both of which most likely have luminosity type II or higher. The system is relatively young, and the fact that the less massive star is located further away from the main sequence implies mass transfer onto the hot companion. The properties of AS 381 (most of the spectral features and SED) are similar to those of MWC 623. The late-type companions of these 2 systems seem to have almost the same fundamental parameters (Zickgraf 2001). However, the hot companion of AS 381 appears to be more massive than that of MWC 623 . We should also note here that AS 381 is not a unique system. Zickgraf et al. (1996) found the LMC B[e] supergiant R4 to be a binary system, which consists of a B- and A-type star. Thus, we can suggest that AS 381 is the first B[e] supergiant binary discovered in the Milky Way.

\section{Conclusions}

We presented and analysed for the first time the observed properties of the emission-line object AS 381. Its spectrum and SED turned out to be similar to those of Be stars with warm dust, a group of nearly 20 galactic peculiar objects. We found that AS 381 is most likely a binary system that consists of two luminous stars of very different spectral types (early-B and K) and is surrounded by a gas-and-dust envelope. The spectral line profiles imply that the envelope is non-spherical and is viewed close to pole-on. Our 2month optical photometric observations did not reveal any noticeable variability, while our near-IR observations and other published near-IR data showed that the object is variable on a time scale of years.

Thus, AS 381 is the third member of this group for which convincing evidence of binarity is found. The other two (MWC 623, CI Cam) also seem to contain at least one luminous stellar component. There are a few more Be stars with warm dust for which the presence of a secondary component is suspected (MWC 342, AS 78, MWC 657). These results give growing evidence that such peculiar strong emission-line objects may be products of the binary evolution at certain stages on or after the main-sequence. However, further systematic observations are needed to make more conclusive statements on the nature of this group. Such observations should include high-resolution spectroscopy to search for $R V$ variations (at least in edge-on systems), optical and near-IR 
Appendix A. Lines identified in the optical spectrum of AS 381.

\begin{tabular}{|c|c|c|c|c|c|c|c|c|c|}
\hline Line ID & $\lambda_{\text {lab }}, \AA$ & $I / I_{\mathrm{c}}$ & $\begin{array}{l}R V \\
\mathrm{~km} \mathrm{~s}^{-1}\end{array}$ & Rem. & Line ID & $\lambda_{\text {lab }}, \AA$ & $I / I_{\mathrm{c}}$ & $\begin{array}{l}R V, \\
\mathrm{~km} \mathrm{~s}^{-1}\end{array}$ & Rem. \\
\hline $\mathrm{H} \gamma$ & 4340.47 & 6.5 & $-42:$ & & Fe I(207) & 6230.73 & 0.85 & -15 & lt \\
\hline$[\mathrm{O}$ III $](2 \mathrm{~F})$ & 4363.21 & 2.8 & $-47:$ & & Fe II $(74)$ & 6238.39 & 1.28: & $-50:$ & \\
\hline He I $(51)$ & 4387.93 & 1.5: & $-43:$ & & Fe II $(74)$ & 6239.91 & - & - & \\
\hline He I(14) & 4471.52 & 1.9: & $-39:$ & & Fe II $(74)$ & 6247.55 & 1.26: & $-50:$ & \\
\hline Fe II (38) & 4508.28 & 1.3: & $-43:$ & & Fe II & 6248.9 & - & - & \\
\hline Fe II $(37)$ & 4515.33 & 1.2: & $-30:$ & & DIB & 6283.85 & $0.56:$ & - & \\
\hline Fe II $(37)$ & 4520.22 & 1.2: & $-48:$ & & {$[\mathrm{O}$ I $](1 \mathrm{~F})$} & 6300.3 & 1.5 & -21 & \\
\hline Fe II(38) & 4522.63 & 1.2: & $-47:$ & & Fe II & 6317.99 & 1.2 & $-43:$ & \\
\hline Fe II(38) & 4549.47 & 1.6: & $-43:$ & & Si II(2) & 6347.10 & 1.26 & $-41:$ & \\
\hline Fe II(37) & 4555.89 & 1.4: & $-35:$ & & Fe I $(13)$ & 6358.70 & $0.84:$ & -20 & lt \\
\hline Cr II(44): & 4558.65 & - & -40 & & {$\left[\begin{array}{ll}\mathrm{O} & \mathrm{I}\end{array}\right](1 \mathrm{~F})$} & 6363.78 & 1.13: & $-28:$ & \\
\hline Fe II(38) & 4583.83 & 1.5: & $-38:$ & & Fe II(40) & 6369.46 & 1.19 & -30 & \\
\hline Fe II(37) & 4629.33 & 1.35: & $-33:$ & & Si II(2) & 6371.36 & 1.25: & $-34:$ & \\
\hline He I(12) & 4713.33 & 1.66: & $-39:$ & & DIB & 6379.29 & 0.85 & - & \\
\hline $\mathrm{H} \beta$ & 4861.33 & 15 & $-42:$ & & Fe II & 6383.72 & 1.17: & $-38:$ & \\
\hline HeI(48) & 4921.93 & 2.5 & $-39:$ & & Fe II & 6385.45 & 1.13: & - & \\
\hline $\mathrm{Fe}$ II $(42)$ & 4923.92 & 1.8: & - & & Fe I $(816)$ & 6400.01 & $0.83:$ & $-18:$ & lt \\
\hline$[\mathrm{O}$ ІІІ $](1 \mathrm{~F})$ & 4958.91 & 3.6 & $-48:$ & & Fe II(74) & 6416.92 & 1.25: & $-41:$ & \\
\hline$[\mathrm{O}$ III] $(1 \mathrm{~F})$ & 5006.84 & 11 & -50 & & Fe II $(40)$ & 6432.68 & 1.38: & $-47:$ & \\
\hline He I $(4)$ & 5015.68 & 2.3 & -41 & & Fe II $(74)$ & 6456.39 & $1.2:$ & $-34:$ & \\
\hline Fe II $(42)$ & 5018.44 & 1.8 & -39 & & Fe II $(40)$ & 6516.08 & 1.45 & -33 & \\
\hline He I(47) & 5047.74 & 1.2: & $-45:$ & & {$[\mathrm{N}$ II] $(1 \mathrm{~F})$} & 6548.03 & 1.3: & $-32:$ & \\
\hline Si II(5) & 5056.1: & 1.3: & $-39:$ & & $\mathrm{H} \alpha$ & 6562.81 & - & -41 & satur. \\
\hline Fe II (42) & 5169.03 & 1.66 & $-42:$ & & {$[\mathrm{N}$ II] $(1 \mathrm{~F})$} & 6583.45 & 2.0 & -41 & \\
\hline Fe II(49) & 5197.58 & 1.5 & $-42:$ & & DIB & 6613.56 & 0.6 : & $-22:$ & \\
\hline Fe II(49) & 5234.62 & 1.49 & $-37:$ & & Ni I(43) & 6643.64 & 0.88 & -30 & lt \\
\hline Fe II(49) & 5276.00 & 1.38: & - & & Fe I(111) & 6663.4 & $0.9:$ & $-12:$ & lt \\
\hline Fe II(41) & 5284.10 & $1.5:$ & -40 & & He I $(46)$ & 6678.15 & 3.37 & -42 & \\
\hline Fe II $(49,48)$ & 5316.61 & 1.88 & -38 & & {$[\mathrm{~S}$ II $](2 \mathrm{~F})$} & 6716.47 & 1.1: & - & \\
\hline Fe II(49) & 5325.56 & 1.25: & - & & $\mathrm{Ca} \mathrm{I}(32)$ & 6717.69 & 0.9 : & $-32:$ & lt \\
\hline Fe II(48) & 5362.86 & 1.44 & $-38:$ & & {$[\mathrm{S}$ II $](2 \mathrm{~F})$} & 6730.85 & 1.16: & $-33:$ & \\
\hline DIB & 5487.67 & 0.86 & - & & Ti I(48) & 6743.13 & 0.89: & -30 & lt \\
\hline Fe II(55) & 5534.83 & $1.5:$ & $-43:$ & & He I $(10)$ & 7065.32 & 4.0 & -39 & \\
\hline $\mathrm{Ca} I(21)$ & 5602.85 & - & $-27:$ & $\mathrm{a}$ & Co I $(54)$ & 7084.98 & 0.88 & -28 & lt \\
\hline Fe I $(686)$ & 5602.95 & - & - & & He I $(45)$ & 7281.35 & 1.78 & $-41:$ & \\
\hline DIB & 5760.40 & $0.9:$ & - & & $\mathrm{Ca}$ I(44) & 7326.16 & 0.87 & $-15:$ & lt \\
\hline DIB & 5762.70 & $0.9:$ & - & & Ni I(109) & 7393.61 & $0.83:$ & -10 & lt \\
\hline DIB & 5766.16 & 0.89: & - & & Fe I(1077) & 7495.08 & $0.85:$ & $-31:$ & lt \\
\hline DIB & 5780.37 & 0.54 & -18 & & Fe I(1077) & 7511.03 & $0.85:$ & $-22:$ & lt \\
\hline DIB & 5785.05 & 0.89: & $-16:$ & & Fe II $(73)$ & 7515.8 & 1.15: & -40 & \\
\hline DIB & 5796.96 & 0.75 & $-20:$ & & Ni I(187) & 7555.61 & - & $-25:$ & lt \\
\hline Ti I(72) & 5866.46 & 0.93: & $-20:$ & lt & Fe I $(402)$ & 7583.80 & 0.86: & $-18:$ & lt \\
\hline He I(11) & 5875.72 & 5.0 & -39 & & Fe I(1137) & 7586.03 & 0.88: & $-7:$ & lt \\
\hline $\mathrm{Na} I(1)$ & 5889.95 & 0.25 & -17 & IS, $-50:^{\mathrm{b}}$ & $\mathrm{K} \mathrm{I}(1)$ & 7664.91 & - & - & IS \\
\hline $\mathrm{Na} I(1)$ & 5895.92 & 0.25 & -17 & IS, $-50:^{\mathrm{b}}$ & $\mathrm{Ni}$ I(62) & 7714.32 & 0.66 : & $-30:$ & lt \\
\hline Fe II $(46)$ & 5991.37 & $1.2:$ & $-40:$ & & $\mathrm{Fe} I(402)$ & 7748.27 & $0.9:$ & -40 & lt ${ }^{\mathrm{c}}$ \\
\hline Fe II $(74)$ & 6147.74 & 1.2: & - & c & Ni I(156) & 7748.90 & - & $-33:$ & lt \\
\hline Fe II $(74)$ & 6149.25 & - & - & & $\mathrm{O} \mathrm{I}(1)$ & 7771.94 & 1.3: & -30 & \\
\hline Ni I(45) & 6191.19 & 0.82 & -11 & lt $^{\mathrm{c}}$ & O I (1) & 7774.17 & - & - & \\
\hline Fe I(169) & 6191.56 & - & $-30:$ & lt & O I $(1)$ & 7775.39 & - & - & \\
\hline DIB & 6195.96 & $0.9:$ & $-25:$ & & Fe I(1154) & 7780.57 & 0.9: & $-22:$ & lt \\
\hline DIB & 6203.08 & 0.86: & $-20:$ & & Ni I(62) & 7788.95 & 0.88 & $-15:$ & lt \\
\hline
\end{tabular}

Comments to the line list in the optical spectrum of AS 381.

Unreliable identifications and less accurate measurements are denoted by colons.

Intensities at line peaks in units of the underlying continuum are given in Col. 3, heliocentric $R V$ in $\mathrm{km} \mathrm{s}^{-1}$ in Col. 4 , and Remarks in Col. 5.

${ }^{\mathrm{a}}$ Blend with the following line, $R V$ relates to both lines; ${ }^{\mathrm{b}} R V$ of the circumstellar component; ${ }^{\mathrm{c}}$ Blend; "satur." - the line top is saturated; "IS" - interstellar feature; "lt" - late-type star feature. 
Appendix B. Lines identified in the near-IR spectrum of AS 381.

\begin{tabular}{llllll}
\hline$\lambda_{\text {lab }}$ & ID & $I / I_{\mathrm{c}}$ & $\lambda_{\text {lab }}$ & ID & $I / I_{\mathrm{c}}$ \\
\hline 8446.35 & O I (4) & 1.37 & 10459.79 & N II $(11):$ & 1.04 \\
8498.018 & Ca II (2) & 1.23 & 10501.00 & Fe II & 1.05 \\
8542.089 & Ca II $(2)$ & 1.20 & 10683.08 & C I & 1.09 \\
8598.394 & P14 & 1.12 & 10830.34 & He I $(1)$ & 4.42 \\
8662.140 & Ca II (2)+P13 & 1.19 & 10938.09 & P6 & 1.30 \\
8750.00 & P12 & 1.12 & 11287.0 & O I & 1.38 \\
8862.787 & P11 & 1.14 & 11750.0 & C I & 1.08 \\
9017.911 & P10 & 1.12 & 12818.05 & P5 & 1.76 \\
9061.33 & Fe II (71) & 1.10 & 16109.31 & Br13 & 1.05 \\
9229.017 & P9 & 1.26 & 16407.19 & Br12 & 1.03 \\
9403.36 & Fe II (71) & 1.11 & 16806.52 & Br11 & 1.04 \\
9545.974 & P8 & 1.18 & 17362.11 & Br10 & 1.05 \\
9997.58 & Fe II & 1.06 & 21655.29 & Br $\gamma$ & 1.10 \\
10049.38 & P7 & 1.27 & & & \\
\hline
\end{tabular}

photometry to search for brightness variations, and far-IR and submillimetric observations to constrain parameters of the objects' dusty environments.

Acknowledgements. A.M. and K.S.B. acknowledge support from NASA grant NAG5-8054. Karen Bjorkman is a Cottrell Scholar of the Research Corporation, and gratefully acknowledges their support. This work was supported in part by a Civilian Recearch and Development Foundation (CRDF) grant RP1-2264. R. O. Gray acknowledges the partial support of a Research Corporation Grant. P. G.-L. acknowledges support from grant PB97-1435-C02-02 from the Spanish Dirección General de Enseñanza Superior e Investigación Científica (DGESIC). V.K. acknowledges support from grant 99-02-18339 of the Russian Foundation for Basic Research. R.J.R., D.K.L., and S.M. were supported by the Independent Research and Development program at The Aerospace Corporation. This research has made use of the SIMBAD database operated at CDS, Strasbourg, France. This publication makes use of data products from the Two Micron All Sky Survey, which is a joint project of the University of Massachusets and the Infrared Processing and Analysis Center, funded by the National Aeronautic and Space Administration and the National Science Foundation.

\section{References}

Aaronson, M., Blanco, V. M., Cook, K. H., Olszewski, E. W., \& Schechter, P. L. 1990, ApJS, 73, 841

Allen, D. A., \& Swings, J.-P. 1976, A\&A, 47, 293

Arribas, S., \& Martínez-Roger, C. 1987, A\&AS, 70, 303

Coluzzi, R. 1993, Bull. Inf. CDS, 43, 7

Dong, Y. S., \& Hu, J. Y. 1991, Chin. A\&A, 15, 275

Dubath, P., Mayor, M., \& Burki G. 1988, A\&A, 205, 77

Egan, M. P., Price, S. D., Moshir, M. M., et al. 1999, The
Midcourse Space Experiment Point Source Catalog Version 1.2, Explanatory Guide, AFRL-VS-TR-1999-1522

Elias II, N. M., Wilson, R. E., Olson, E. C., et al. 1997, ApJ, 484,394

Herbig, G. H. 1993, ApJ, 407, 142

Hinkle, K., Wallace, L., Valenti, J., \& Harmer, D. 2000, Visible and Near Infrared Atlas of the Arcturus Spectrum 37279300 A (San Francisco: ASP)

Humphreys, R. M. 1978, ApJS, 38, 309

Kurucz, R. L. 1994, Smithsonian Astrophys. Obs., CD-ROM No. 19

Lamers, H. J. G. L. M., Zickgraf, F.-J., de Winter, D., Houziaux, L., \& Zorec, J. 1998, A\&A, 340, 117

Merrill, P. W., \& Burwell, C. G. 1933, ApJ, 78, 87

Merrill, P. W., \& Burwell, C. G. 1950, ApJ, 112, 72

Miroshnichenko, A. S. 1997, In Fundamental Stellar Parameters, Proc. IAU Symp. 189, ed. T. R. Bedding (Publ. School of Physics, Univ. of Sydney, Australia), 50

Miroshnichenko, A. S. 1998, In B[e] stars, Ap\&SS Library, 233, 145

Miroshnichenko, A. S., \& Corporon, P. 1999, A\&A, 349, 126

Miroshnichenko, A. S., Ivezić, Ž., Vinković, D., \& Elitzur, M. 1999, ApJ, 520, L115

Miroshnichenko, A. S., Chentsov, E. L., Klochkova, V. G., et al. 2000, A\&AS, 147, 5

Miroshnichenko, A. S., Levato, H., Bjorkman, K. S., \& Grosso, M. 2001, A\&A, 371, 600

Panchuk, V. E., Najdenov, I. D., Klochkova, V. G., et al. 1998, Bull. Spec. Astrophys. Obs., 44, 127

Rudy, R. J., Erwin, P., Rossano, G. S., \& Puetter, R. C. 1991, ApJ, 383, 344

Rudy, R. J., Puetter, R. C., \& Mazuk, S. 1999, AJ, 118, 666

Schaller, G., Schaerer, D., Meynet, G., \& Maeder, A. 1992, A\&AS, 96, 269

Sheikina, T. A., Miroshnichenko, A. S., \& Corporon, P. 2000, in The Be-phenomenon in Early-Type Stars, ed. M. A. Smith, H. F. Henrichs, \& J. Fabregat, ASP Conf. Ser., 214, 494

Shevchenko, V. S. 1989, Herbig Ae/Be stars, Tashkent, Fan

Skrutskie, M. F., Schneider, S. E., Stiening, R., et al. 1997, in Proc. Workshop, The Impact of Large Scale Near-IR Sky Surveys, 25

Stock, J., Nassau, J. J., \& Stephenson, C. B. 1960, Luminous Stars in the Northern Milky Way, Part II

Thé, P. S., de Winter, D., \& Peréz, M. R. 1994, A\&AS, 104, 315

Wackerling, L. R. 1970, Mem. RAS, 73, 153

Wallace, L., \& Hinkle, K. 1997, ApJS, 111, 445

Wallace, L., Meyer, M. R., Hinkle, K., \& Edwards, S. 2000, ApJ, 535, 325

Waters, L. B. F. M., Coté, J., \& Lamers, H. J. G. L. M. 1987, A\&A, 185, 206

Weaver, Wm. B., \& Jones, G. 1992, ApJS, 78, 239

Zickgraf, F.-J., \& Stahl, O. 1989, A\&A, 223, 165

Zickgraf, F.-J., Kovács, J., Wolf, B., Stahl, O., Kaufer, A., \& Appenzeller, I. 1996, A\&A, 309, 505

Zickgraf, F.-J. 2001, A\&A, 375, 122 